In Crescendo. Institucional. 2015; 6(1): 157-166

\title{
ESTRATEGIAS DE ORIENTACIÓN FUNDAMENTADAS EN TÉCNICAS DE COACHING ONTOLÓGICO PARA MEJORAR LA CALIDAD COMUNICACIONAL ENTRE LOS DOCENTES
}

\section{ORIENTED STRATEGIES GROUNDED IN ONTOLOGICAL COACHING TECHNIQUES TO IMPROVE COMMUNICATION QUALITY AMONG TEACHERS}

Luis Enrique Vásquez Giménez*

\begin{abstract}
RESUMEN
E propósito de la siguiente investigación es proponer estrategias de orientación fundamentadas en técnicas de coaching ontológico para mejorar la calidad comunicacional entre los docentes del colegio San José de Tarbes de La Florida, Caracas. Es una investigación cualitativa, con un diseño emergente, apoyada en una metodología interpretativa fenomenológica. Se tomaron como informantes claves a nueve docentes que laboran en la referida institución educativa. Se aplicaron las técnicas de la entrevista y la observación participante, y sus respectivos instrumentos, el guión de entrevista y el registro anecdótico. La información se validó a partir de lo que pudo aflorar de los mismos informantes claves a partir de la triangulación de las técnicas, la transferibilidad y lo argumentativo. Se describen las categorías de análisis que surgieron como producto de los insumos obtenidos y, finalmente, se diseñan las estrategias de orientación fundamentadas en técnicas de coaching ontológico. Se obtuvo como resultado que las estrategias de orientación formuladas permiten a los docentes observarse, pensar, sentir y comunicarse de manera efectiva y afectiva; y, paralelamente, redimensionar sus redes conversacionales con el fin de mejorar sus competencias comunicativas dentro de esta organización educativa. Como conclusión, se señala que las estrategias de orientación expresadas en este trabajo llevan a desarrollar las formas de ser que permitan lograr el cambio a los docentes operando sobre su experiencia comunicacional y de relaciones intra e interpersonales.
\end{abstract}

\footnotetext{
* tobit7@hotmail.com
} 
Palabras claves: estrategias de orientación, técnicas de coaching ontológico, calidad comunicacional.

\begin{abstract}
The purpose of this research is to propose guidance strategies grounded in ontological coaching techniques to improve communication quality among teachers of Colegio San José de Tarbes of La Florida, Caracas. It is a qualitative research, with an emergent design, based on a phenomenological interpretative methodology. They were taken as key informants to nine teachers who work in said school. Interview techniques and participant observation, and their instruments, the script interview and anecdotal record is applied. The information was validated from what might emerge from the same key informants from triangulation techniques, portability and argumentative. The categories of analysis that emerged as a result of inputs obtained and finally guidance strategies grounded in ontological coaching techniques described are designed. It resulted that made orientation strategies allow teachers to observe, think, feel and communicate effectively and affectively; and, in parallel, they resize your conversational networks in order to improve their communication skills in this educational organization. In conclusion, it is noted that guidance strategies expressed in this work lead to develop ways of being that would achieve the change to teachers operating on their experience and intra communication and interpersonal relationships.
\end{abstract}




\section{INTRODUCCIÓN}

El proceso de comunicación es el elemento fundamental en cualquier organización, pues de ello se deriva el fomento de las relaciones, el flujo de informaciones que permite el logro de los objetivos de la institución y, por consiguiente, propicia buen clima organizacional, según lo expresa Gibbson (1996). De acuerdo a lo planteado, es en el ámbito educativo donde la comunicación actúa y propicia un proceso de estudio sobre el comportamiento de todas las personas involucradas en el contexto organizativo, ya que en cada organización educativa existe una estructura y lineamientos formales que deben ser cumplidos sin necesidad de utilizar normas rígidas que ocasionan el desinterés y la apatía del personal. Es por ello que de la calidad de ese vínculo depende, en gran medida, de la calidad de las relaciones de sus miembros.

La comunicación como parte vital de toda organización educativa, donde la interacción es propiciada por las relaciones interpersonales, por el contacto que se establece entre las personas, en diferentes contextos e intervalos de tiempo. Es así que la comunicación eficaz constituye la parte esencial en el desarrollo de la capacidad, motivación y productividad entre los miembros de una organización educativa, para mejorar los niveles de efectividad y eficiencia de las mismas.

De allí que, es importante considerar a la organización como una alternativa para el desarrollo personal en grupo. De esta manera, el desarrollo organizacional tiene gran influencia en la eficiencia de una colectividad, por cuanto genera cambios de actitud en cada uno de sus miembros, lo cual se traduce en rendimiento para el logro de las metas comunes. El recurso humano es clave para el éxito de la institución; en consecuencia, es fundamental su conducción adecuada y el desarrollo de relaciones humanas que permitan prevenir conflictos.

Por tanto, la propuesta de diseñar estrategias de orientación fundamentadas en técnicas de coaching ontológico para mejorar la calidad comunicacional entre los docentes del Colegio San José de Tarbes de La Florida, Caracas, aportará una mejor comprensión desde un sentido humanista que lleve a valorar la peculiaridad de las personas, reconociendo que cada ser humano es diferente. Propiciando la toma de conciencia, que es la que otorga libertad y capacidad de elección a la persona, siendo uno de los principios del Programa del Sistema Nacional de Orientación (PSNO).

Para Dezerega (2008), el coaching ontológico es un método de aprendizaje de desarrollo personal y profesional, basado en la capacidad de accionar y de conseguir resultados basados en la eficacia y el bienestar. Perry y Skiffington (2002), plantean que el objetivo del coaching ontológico es permitir que las personas den lo mejor de sí mismas, para ello utiliza la formación y desarrollo de habilidades como el liderazgo de un equipo de trabajo, 
orientación a la persona, mejora de la eficacia, adaptaciones a situaciones nuevas, la ayuda para la toma de decisiones o la superación de dificultades.

\section{PROBLEMA}

¿Qué herramientas de desarrollo personal manejan los docentes para afrontar sus procesos comunicacionales y de relaciones interpersonales?

\section{OBJETIVO GENERAL}

Proponer estrategias de orientación fundamentadas en técnicas de coaching ontológico para mejorar la calidad comunicacional entre los docentes del Colegio San José de Tarbes de La Florida.

\section{OBJETIVOS ESPECÍFICOS}

1. Describir las herramientas que utilizan o tienen los docentes para afrontar sus procesos comunicacionales y de relaciones interpersonales.

2. Caracterizar las dimensiones del desarrollo humano en cuanto a comunicación y relaciones interpersonales que favorezcan el acompañamiento entre los docentes.

3. Construir un cuerpo de estrategias de orientación para optimizar la calidad comunicacional y de relaciones interpersonales de los docentes basadas en técnicas de coaching ontológico.

\section{MATERIAL Y MÉTODOS}

Esta investigación, inmersa en el contexto educacional, se abordó desde una metodología cualitativa. Siguiendo a Sandín (2003), la investigación cualitativa en educación se define como: «una actividad sistemática orientada a la comprensión en profundidad de fenómenos educativos y sociales (...) y también orientada hacia el descubrimiento y desarrollo de un cuerpo organizado de conocimientos» (p. 123). También Martínez, (citado por Hurtado, 2008), al referirse a esta, afirma: "Se centra en la importancia del cambio, el contexto, la función y el significado de los actos humanos y no pretender hacer generalizaciones universales, sino estudiar, conocer y servir a una comunidad, adoptando inicialmente una actitud exploratoria y de apertura mental para comprender las realidades tal como existen, y describirlas con riqueza de detalles." (p. 7). 
Como se puede apreciar, tanto en la definición de Sandín como en lo expuesto por Martínez, más que resaltar el tipo de procedimiento metodológico, se pretende destacar la finalidad con la que el investigador aborda la temática. En el caso de la presente investigación el propósito fue la interpretación de los hallazgos y comprender la realidad del problema planteado.

Según Lincoln (citado por Sandín, 2003, p. 29), "la investigación cualitativa debe responder a tres cuestiones básicas: ontológica, epistemológica y metodológica". Desde la dimensión ontológica (naturaleza de la realidad), se percibe la realidad como una construcción del investigador y los participantes, donde todos los elementos que intervienen están conectados entre sí; en este caso se trató de las relaciones comunicacionales e interpersonales entre los docentes y el contexto donde se desarrollan.

Desde la dimensión epistemológica, la intención fue construir significados de la experiencia, de la realidad de las relaciones comunicacionales e interpersonales entre los docentes. Se desarrolló mediante la interacción entre el autor de esta investigación y los participantes de una forma directa y dialógica, de manera tal que se logró una vivencia acerca de la realidad sujeta de estudio, lo cual permitió comprenderla. A la vez que posibilitó a los docentes describir la realidad desde sus propias vivencias y experiencias, objetivando el mundo tal como lo viven y lo experimentan cotidianamente en su entorno sociocultural. Los docentes elaboraron descripciones sobre su vida cotidiana y expresaron el universo de códigos y representaciones que los constituyen. En este sentido, se habla de la dimensión epistemológica que emergió en cuanto a conocimiento de sí mismo y aprehensión de su realidad visto que proporcionaron información sobre cada uno, sus prácticas, sus relaciones cotidianas, percepciones, relatos e historias. Los docentes de manera individual y colectiva identificaron, reconocieron, descifraron y describieron sus modos de sentir, pensar, actuar, imaginar y creer relacionándolos con otros sujetos, escenarios, eventos y dinámicas sociales, lo cual es plasmado y representado de forma gráfica, verbal, textual y dramática.

Dicha interacción permitió también la creación de un conjunto de conocimientos cargado de valores, creencias, costumbres, sentimientos y otros aspectos que forman un todo en la persona del investigador. Esto le dio cierto carácter subjetivo a la realidad, resaltando lo humano y vivencial de la experiencia.

En cuanto a la dimensión metodológica, se asumió un camino flexible donde la interrelación con los participantes se convirtió en momento oportuno para propiciar el diálogo entre ellos y con ellos.

Tal como se evidencia en las entrevistas realizadas y en la observación participante, desde el diálogo y la relación generada entre el investigador y los docentes, se logró que el grupo se acercara a comprender y explicar el sentido de lo expresado por ellos mismos, haciendo una lectura comprensiva y problematizadora, desde sentidos personales, históricos y culturales. De expresiones vitales significativas en el quehacer de las personas en sus diversos escenarios de actuación, pero con arraigo en su contexto originario. Se buscó ubicar, orientar, explicar y recuperar los saberes y las experiencias que están detrás de lo que han plasmado y manifestado los docentes participantes, estableciendo relaciones que 
llevan a otros sentidos, teniendo en cuenta que los signos, símbolos, códigos y lenguajes los ubican en una temporalidad que corresponde a movimientos históricos y costumbres culturales, que llevaron a emprender tránsitos hacia realidades invisibles, latentes, profundas, plenas de sentidos. Posibilitando comprender otras realidades a la luz de lo que son como sujeto.

En síntesis, de la interpretación fenoménica de este estudio, emergieron cuatro dimensiones filosóficas pragmáticas que se ilustran a continuación:

Para el estudio investigativo se usó la técnica de entrevista con su respectivo instrumento, la guía de entrevista, que corresponde a una forma estructurada de obtener información. Su carácter profundo permite realizar un sondeo detallado de los sentimientos, opiniones, creencias y actitudes del sujeto seleccionado expresado en su propio lenguaje;

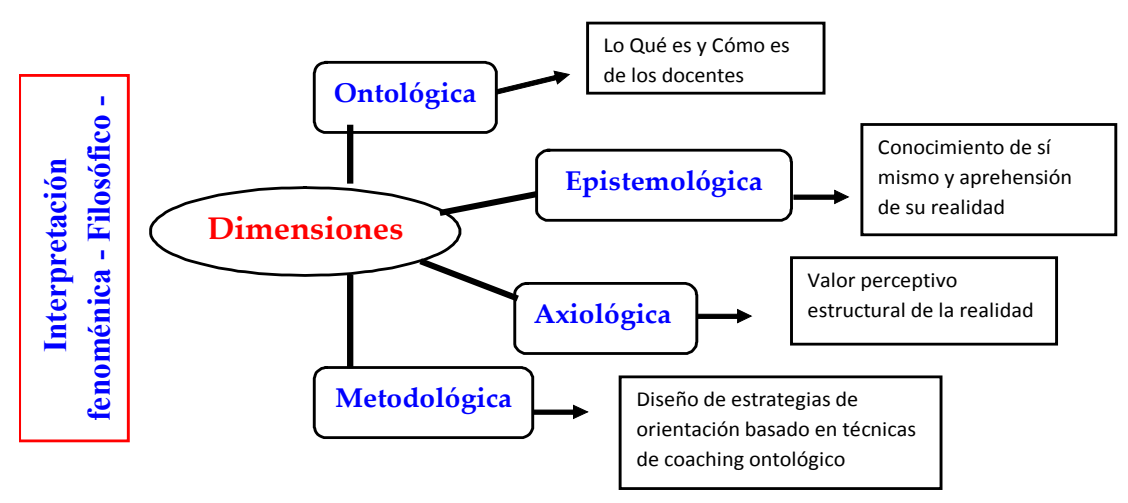

Gráfico 1. Síntesis - Interpretación Fenoménica - Filosófica -Pragmática

Fuente: Vásquez (2012).

además, proporciona datos para hacer un análisis de temas confidenciales, delicados o suspicaces en los que el informante podría tener o no la influencia del grupo.

En segundo lugar, se seleccionó como técnica la observación participante, con su respectivo instrumento, el registro de observación, ya que esta implica que el investigador o el responsable de recolectar la información se involucre directamente con la actividad objeto de la investigación, lo que puede variar desde la integración total al grupo o ser parte de este durante un periodo. La triangulación de ambos métodos permitirá una mayor profundidad y riqueza de la información, y revestirá de validez a la investigación.

Para efectos de este estudio investigativo, se asume como método para la interpretación de los resultados la triangulación de las técnicas e instrumentos que se aplicaron: la entrevista estructurada (Guión de entrevista) general que fue aplicada a los directores, subdirectores, a los docentes de cada etapa que fueron seleccionados según los siguientes criterios: tiempo laborando en la institución, participación en actividades planificadas por el colegio. Observación participante (Registro anecdótico) a través de la entrevista 
grupal, el cual estuvo conformado por nueve (9) informantes claves, es decir, dos (2) docentes de cada una de los niveles del subsistema de educación básica, inicial, primaria, media diversificada. A través de la entrevista grupal, se extrajeron insumos informativos para complementar los reportes obtenidos en las respuestas de las entrevistas. Todas las informaciones obtenidas por medio de la aplicación de las diferentes técnicas se fueron triangulando hasta saturarla y de ellas obtener elementos teóricos básicos y concretos que condujeron al diseño de las estrategias de orientación fundamentadas en técnicas de coaching ontológico.

En este estudio se atiende a la formulación de Rodríguez, Gil y García (1999) quienes indican que analizar los datos cualitativos "se refiere a tratamientos de los datos que se llevan a cabo generalmente preservando su naturaleza textual, poniendo en práctica, tareas de categorización y sin recurrir a técnicas estadísticas".

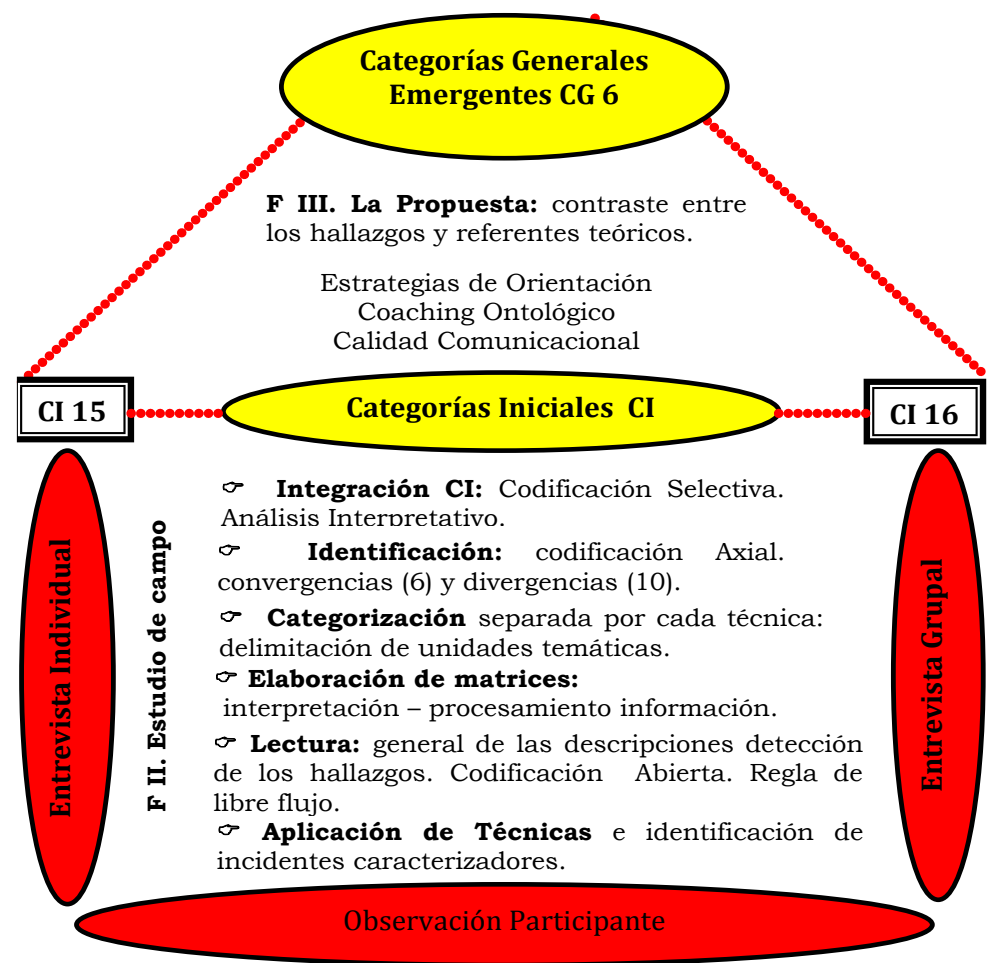

F I. Planeación. Exploración de fuentes. Referentes teóricos.

Gráfico 2. Fases de la aplicación del método fenomenológico

Fuente: Vásquez (2012)

\section{RESULTADOS}

La investigación posibilitó a los docentes como participantes claves, reflexionar sobre la vivencia durante el desarrollo de las técnicas a nivel individual y grupal; la manera 


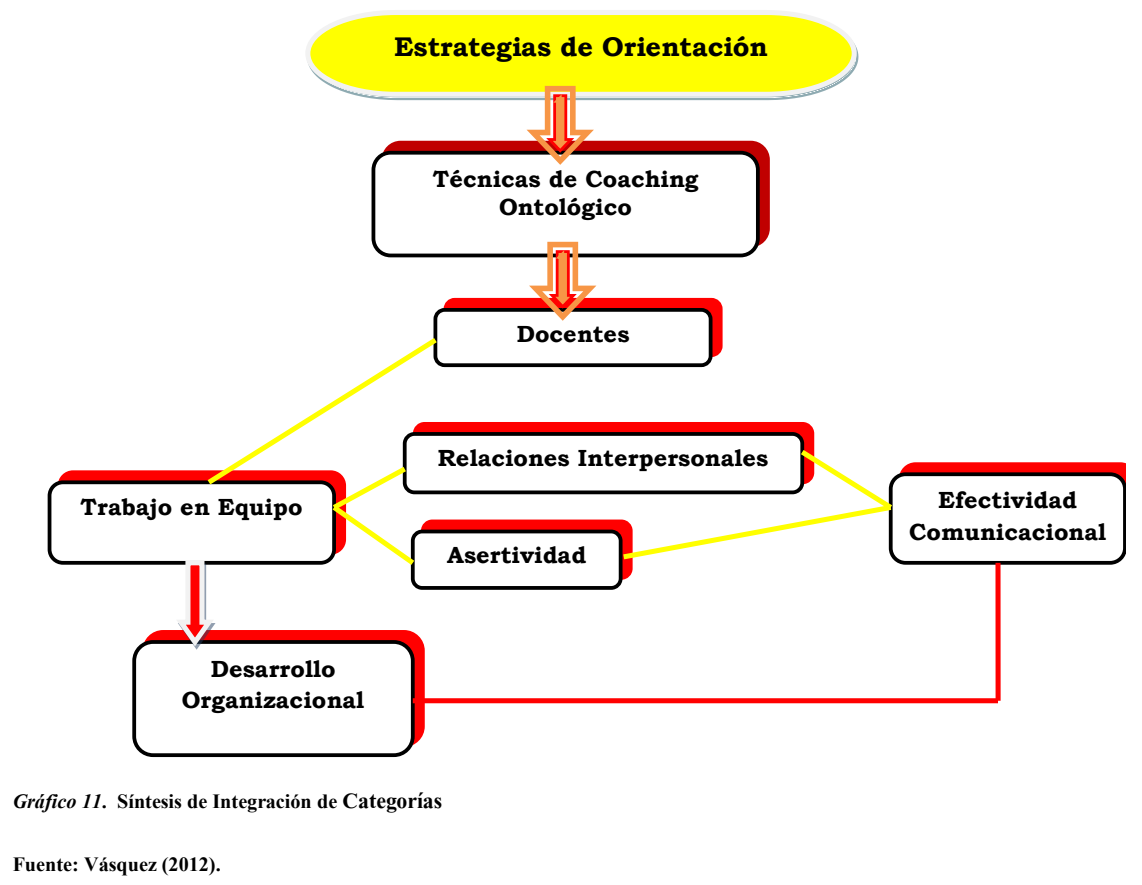

cómo se dio la comunicación, la capacidad de escucha y la comprensión del tema, las dificultades que se presentaron, la forma en que se sintió cada persona y los aspectos que facilitaron o no la participación y la expresión. De manera que llevó a los docentes a construir un conocimiento más profundo de su realidad individual y social, lo cual les posibilitó comprenderla y ubicarse en ella; y, de esta manera, orientar acciones hacia su transformación, dando pistas para plantear y replantear otras formas de relacionarse, de deconstruir y resignificar vivencias, saberes y sentidos.

\section{DISCUSIÓN}

Tomando en consideración que la ontología es la parte de la metafísica que estudia el Ser en general y sus propiedades trascendentales (Ferrater, 2001). También puede llamarse como el estudio del Ser en tanto lo qué es y cómo es. La ontología define al Ser y establece las categorías fundamentales de las cosas a partir del estudio de sus propiedades, sistemas y estructuras. Por tanto, desde la propuesta se plantea una dimensión ontológica que pretende comprender al sujeto en cuanto a lo qué es y cómo es, en consecuencia las técnicas se estructuran de la siguiente manera: 1. Nombre de la técnica, 2. Objetivo, 3. Características, 4. Ciclo de desarrollo: 4.1 Descripción, 4.2. Expresión, 4.3. Interpretación, 4.4. Toma de conciencia y 4.5. Evaluación.

El diseño de las estrategias basadas en técnicas, según lo plantea Dezerega, (2008), se han clasificado en: descriptivas, histórico-narrativas, analíticas y expresivas. 
Descriptivas: Son aquellas técnicas que a partir del dibujo, de la pintura, de la escritura, del retrato o de la fotografía, permiten que los sujetos plasmen y narren situaciones, hechos, momentos, ambientes, dando cuenta de los diferentes componentes y características de esa realidad, al igual de las diferentes comprensiones y percepciones que los sujetos tienen de ella. Al utilizar técnicas descriptivas se busca que las personas expresen el mundo tal y como lo viven y lo experimentan cotidianamente, donde el lenguaje como texto social, permite la expresión y el desciframiento de modos de sentir, pensar, actuar y relacionar.

Histórico-Narrativas: Estas técnicas tienen un interés por rescatar la experiencia de los sujetos y la recuperación de procesos, hechos, acontecimientos que se dieron en el pasado, pero que cobran vida en el presente e inciden en el futuro; al utilizar estas técnicas, se busca la interpretación y comprensión de esas vivencias y hechos, teniendo en cuenta el contexto en el que se desarrollaron.

Analíticas: Este tipo de técnicas llevan a los participantes a reflexionar sobre problemas sociales, sus propias vidas y la realidad social que habitan, estableciendo relaciones y cruces entre los aspectos que se evidencian y haciendo tránsitos y comparaciones que nos lleven hacia la comprensión de situaciones reales, de la sociedad y de la vida misma. Facilitan la comprensión y explicación de los sentidos que se encuentran ocultos detrás de la cotidianidad de las acciones e interacciones de los sujetos con su entorno, posibilitando el hacer evidente las causas que motivan a los actores de una realidad a interactuar en ella, a habitarla y a configurarla de una manera determinada.

Expresivas: Son técnicas que se caracterizan por hacer posible que los sujetos manifiesten sus sentimientos y pensamientos; para ello, se valen de manifestaciones gestuales, orales, escritas, musicales y plásticas; en ellas, las personas elaboran relatos sobre el mundo de la vida y ponen a prueba signos y símbolos que permiten el acercamiento comprensivo y la expresión de sus formas de constituirse como sujetos sociales.

\section{CONCLUSIONES}

La importancia de promover un proceso de capacitación donde los docentes puedan comprender y manejar las experiencias presentes que explican quiénes son, clarificando los sistemas de valores y creencias asumidos para reconstruir nuevas formas de escuchar, ver, sentir y pensar el mundo; es decir, formas de percibir su propio mundo personal e interpersonal y, por ende, de quienes les rodean, todo lo cual redundó en la apertura hacia la construcción de nuevas redes de relaciones interpersonales a intragrupales dentro de quienes laboran en la institución educativa.

El seleccionar y proponer estrategias de orientación fundamentadas en técnicas de coaching ontológico, se dirigió precisamente a mejorar los resultados de su trabajo como docente a corto plazo y para resolver problemas desde su cotidianidad. El fundamentarse 
en técnicas de coaching ontológico, implica que estas trabajan sobre un tipo de observador que somos de la realidad. Supone que cada uno de nosotros tiene una experiencia del mundo que es coherente con nuestra manera de actuar y al accionar de manera congruente desde los dominios del lenguaje, lo emocional y lo corporal se abren espacios de sintonías interpersonales más auténticas y próximas en el verdadero contacto humano desde la mismidad con la otredad y su impacto en la cotidianidad.

Por tanto, las estrategias de orientación formuladas en este trabajo llevaron a desarrollar las formas de ser adecuadas que permitan lograr objetivos. En este sentido, la clave de alcanzar lo que se propone está en aprender a ser de la manera que hay que ser para lograrlo. Es el proceso por el cual se enseña el cambio a las personas operando sobre su experiencia y no sobre sus conceptos.

\section{REFERENCIAS BIBLIOGRÁFICAS}

Dezerega, V. (2008). Apuntes de gerencia estratégicas en un contexto de coaching integral de negocios. Caracas: IESA.

Ferrater, Mora (2001). Diccionario de filosofía. Madrid: Ariel.

Gibbson, I. (1996). Las organizaciones: comportamiento, estructura y proceso. México: McGraw-Hill/Interamericana.

Martínez, M. (2009). Ciencia y arte en la metodología cualitativa. México: Trillas.

Perry, Z. y Skiffington, S. (2002). Guía completa de coaching en el trabajo. México: McGraw-Hill Interamericana.

Proyecto Sistema Nacional de Orientación (2008). Ministerio Popular para la Educación Superior. Caracas.

Rodríguez, G., Gil, J. y García, E. (1999). Enfoques en la investigación cualitativa. Metodología de la investigación cualitativa. Málaga: Aljibe.

Sandín, M. (2003). Investigación cualitativa en educación. Barcelona: McGraw-Hill. 